\title{
Flexible metamaterials for advanced photonics applications
}

\author{
P. Reader-Harris, M. Pietrzyk, Y. Shen, B. Kirkpatrick and A. Di Falco \\ University of St Andrews, School of Physics and Astronomy, North Haugh, KY16 9SS, St Andrews, UK \\ adf10@st-andrews.ac.uk
}

\begin{abstract}
We present our recent results on the design, fabrication and applications of flexible metamaterials in the visible and near infrared range. In particular, we demonstrate broad angle spectral filtering for lab-on-fibers applications, the realisation of metamaterials with epsilonnear-zero response and their dynamical tuning via all-optical methods.
\end{abstract}

\section{INTRODUCTION}

Flexible metamaterials retain the potential of nanoplasmonics to concentrate the optical field at the nanoscale, the versatility in design typical of metameterials [SoukoulisNegative indexflexible] and metasurfaces [APLDifalcoAlu] and the advantages of having supple substrates. The ability to modify the shape of the substrate allows functionalizing complex shaped object [PJRH,SimonHorsley]. This allows to decouple the fabrication requirements of the metamaterial from the specific shape of the target substrate. This is a crucial advantage given the inherent difficulty in realising metallic nanometric features in non-planar geometries, as required by typical MMs in the visible range. Flexibility additionally offers post-fabrication tuning possibilities [Baumberg,Atwater] which can also be combined with all-optical methods, e.g. resorting to nonlinear optical effects [RizzaAPL]. In this paper we report on the fabrication routes to flexible metasurfaces and epsilon near zero metamaterials in the visible range as example of the conformability and tunability. We also discuss our progress in the optical manipulation of the flexible samples via optical tweezer, to achieve extremely accurate mechanical control of the membranes.

\section{FABRICATION}

In figure 1 we show a procedure to fabricate flexible metasurfaces (left panels) and epsilon near zero metamaterials (right panels). In both cases we first spin a polymeric sacrificial layer. The polymer is chosen such as to be orthogonal to the rest of the fabrication, i.e. ensuring compatibility with the temperature and the solvents used for the following steps. In the case of the metasurfaces, a support polymeric membrane of the required thickness is then spun and cured. Potential polymers include SU8, polystyrene or polymethyl methacrylate (PMMA), with thicknesses varying from few tens $\mathrm{nm}$ to several tens of microns. To realize the metallic nano features we deposit a $40 \mathrm{~nm}$ thick gold layer and then use electron beam lithography to write on a resist of choice, either negative (e.g. SU8 or Hydrogen silsesquioxane) or positive (e.g. PMMA). After development the pattern is typically transferred onto the gold with an Argon based reactive ion etch step. The membrane is eventually released from the substrate by dissolving the sacrificial layer via immersion in a suitable solvent. To realise the epsilon near zero metamaterials we deposit on top of the sacrificial layer polymers and metals of the required thickness. The rule of thumb is to try and minimse the thickness of the metal as much as possible, to minimise absorption losses. The polymer thickness is then calculated accordingly to realise the epsilon near zero condition (see e.g. Salandrino, Rizza). A critical issue to consider is that often the metal does not wet easily the polymeric substrate, thus forming isolated droplets instead of a thin continuos film. This feature can be remedied for example by gently ashing with oxygen plasma the polymer before every metal deposition. Once the required number of layers is reached the whole multilayer is removed from the substrate by dissolving the sacrificial layer.

\section{RESULTS}

Flexible metasurfaces are especially well suited for lab-on-fiber applications, where a photonic function is implemented directly on the tip of the fiber. Typical approaches consist on direct writing of the fiber's facet, e.g. via 
focused ion beam or electron beam lithography [refs] or on the transfer of metallic nano features [Capasso]. Our approach consist on wrapping the membrane on the tip of the fiber [PJRH]. This is convenient because the fabrication of the nano pattern does not require manipulating precisely the finer and also because the photonic function can be replaced cheaply, without the need to change the finer itself. Most importantly, our method is the only one that allow functionalising hollow-core photonic crystal fibers (HPhCF), since direct methods are obviously not suitable. Fig. 1d) shows the experimental transmission spectra of a HPhCF for different polarisations. In this case the metasurfaces implements a broad-angle spectral filter obtained engineering the guided more resonance of the membrane ( $\simeq 250 \mathrm{~nm}$ thick) and a metallic grating with period $600 \mathrm{~nm}$ and mark-to-space ratio equal to 0.25 . The insets of the panel show a Scanning electron microscope image of the tip of the HPhCF and a sketch of the membrane wrapped on the fiber. Epsilon near zero metamaterials could also be useful for lab-on-fiber applications, although they are mostly studied in different context. For example they are at the basis of imaging devices with resolving power beyond the diffraction limit [Salandrino]. The flexibility could be used in this context to tune the property of the lens. To reach this goal a crucial limiting factor is the propagation loss due to the metal layers. In a recent work we have shown that a potential solution would be that of adding a dye to the polymer to compensate for the losses [Rizza]. Fig 1g) shows the absolute value of the retrieved perimittivity with and without external pumping, showing a reduction of $20 \%$ on its minimum value. This curves were obtained on the sample before releasing the membrane. The inset of the figure shows a flexible realisation. Measurements on the tuning properties will be reported elsewhere.

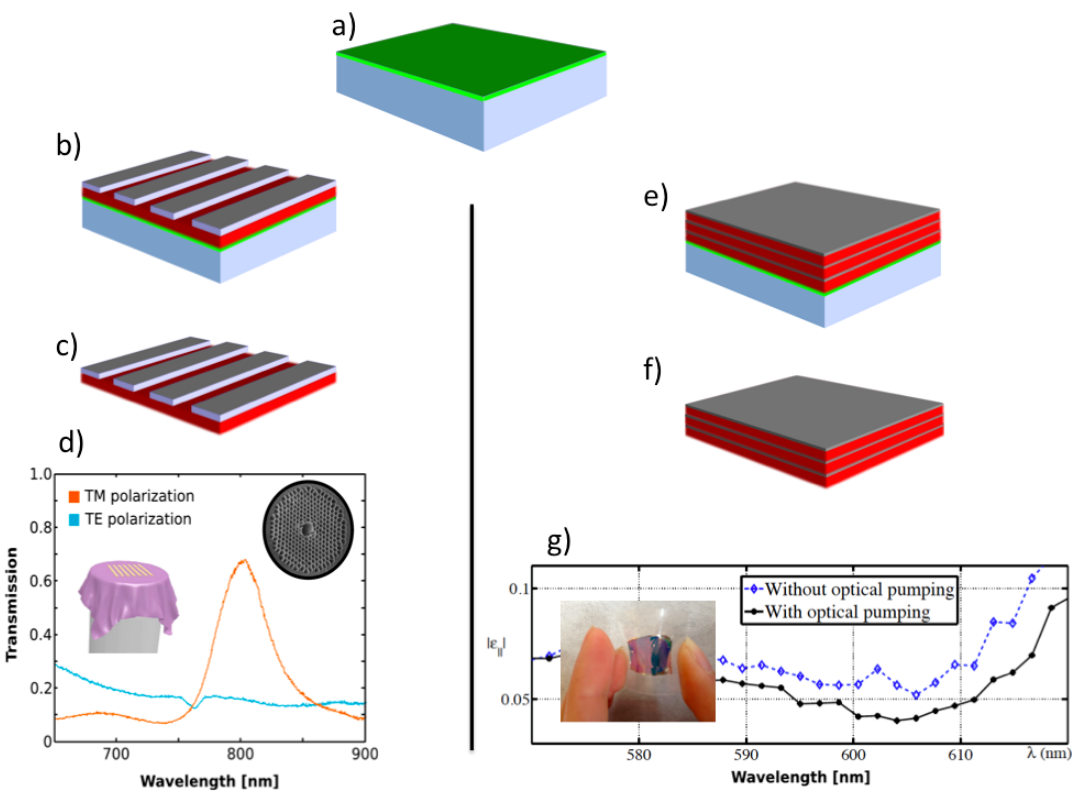

Fig. 1: fig1

\section{OPTICAL MANIPULATION}

While it is possible to control macroscopically the shape and the position of the flexible membrane, it is desirable to have a control at microscopic level as well, if not below. To achieve this goal we have developed an optical tweezer setup that allow to precisely adjust the position in time and space of large photonic membranes. The optical setup consists of a diode-pumped solid state laser $(\lambda=830 \mathrm{~nm})$ expanded with a telescope and focused onto the sample by a $\times 60$ objective lens (Olympus UPLSAPO $60 \mathrm{XW}, \mathrm{NA}=1.2$ ). The use of dichroic mirror and infrared filter allows the user to decide whether or not a small reflection of the laser light from the sample can be projected onto the camera.In this way one may position the optical traps as desired, then place the filter in the path of beam, allowing particle behaviour to be observed without the signal to the camera being dominated by the light from the laser. As shown in fig. 2a-c) non-symmetric objects like a $600 \mathrm{~nm}$ thin membrane of transversal dimen- 
sions $5 x 5 \mu m^{2}$ tend to orient themselves in response to the forces induced by the optical field. Our preliminary results however show that it is possible to stably trap geometries with even more extreme form factors, without reorientation, for example by texturing the membrane surface (see e.g. fig $2 \mathrm{~d}-\mathrm{f}$ )). In this case a $600 \mathrm{~nm}$ thick and $100 x 100 \mu \mathrm{m}^{2}$ membrane was trapped using thicker portion of the membrane as handles.
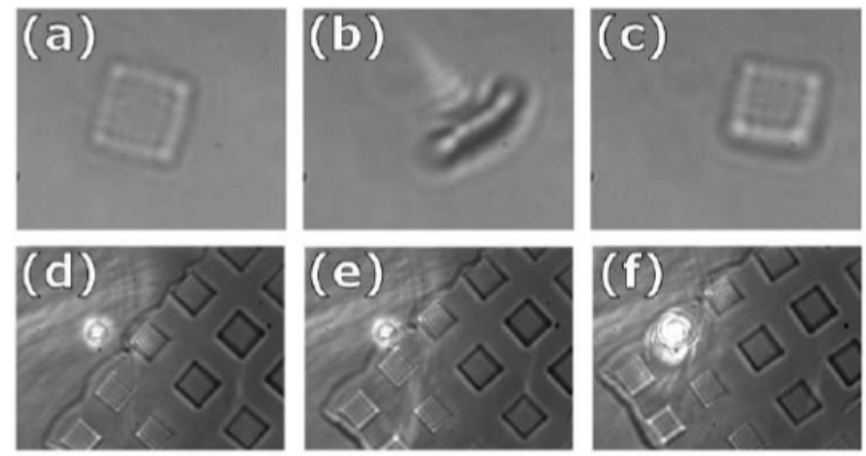

Fig. 2: fig2

\section{CONCLUSiON}

In conclusions we have presented and discussed routes for the fabrication of flexible metamaterials and metasurfaces in the visible range and shown potential uses for lab-on-fiber applications and epsilon near zero materials. We also presented our progress on the all-optical manipulation of flexible membranes via optical tweezers.

\section{ACKNOWLEDGEMENT}

We thank Audrey Thomson, Daniel Mayoh and Sathya Sai Seetharaman for early involvement in the fabrication of epsilon near zero metamaterials and Mathivanan Damodaran for contribution on the optical manipulation experiments. ADF acknowledges support from EPSRC (EP/I004602/1 and EP/J004200/1).

\section{REFERENCES}

[1] C.R. Simovski and S.A. Tretyakov, "Local constitutive parameters of metamaterials from an effective-medium perspective," Physical Review B, vol. 75, p. 195111, 2007.

[2] S.A. Tretyakov and I.S. Nefedov, "Field-transforming metamaterials," Proceedings of Metamaterials'2007, pp. 474-477, Rome, Italy, 22-24 October 2007.

[3] L.D. Landau and E.M. Lifshits, Electrodynamics of Continuous Media, 2nd edition, Oxford, England: Pergamon Press, 1984. 